\title{
Ectomycorrhizal fungus (Paxillus involutus) and hydrogels affect performance of Populus euphratica exposed to drought stress
}

\author{
Zhi-Bin LUO ${ }^{1,2 *}, \mathrm{Ke} \mathrm{LI}^{3}$, Xiangning JiAnG ${ }^{3}$, Andrea Polle ${ }^{1}$ \\ ${ }^{1}$ Büsgen-Institut, Abteilung: Forstbotanik und Baumphysiologie, Georg-August Universität, Büsgenweg 2, 37077 Göttingen, Germany \\ ${ }^{2}$ College of Life Sciences, Northwest A\&F University, Yangling, Shaanxi, 712100, P.R. China \\ ${ }^{3}$ College of Life Sciences and Biotechnology, Beijing Forestry University, Beijing, 100083, P.R. China
}

(Received 30 June 2008; accepted 27 October 2008)

\author{
Keywords: \\ drought / \\ hydrogel / \\ mycorrhizal fungus / \\ poplar / \\ carbohydrate
}

\begin{abstract}
- Mycorrhizal fungi and hydrogels (water-absorbing polymers) can improve water availability for trees. The combination of both factors for plant performance under water limitation has not yet been studied.

- To investigate the influence of the ectomycorrhizal fungus Paxillus involutus, hydrogel and the combination of both factors, a drought-sensitive poplar, Populus euphratica, was examined in this study. - After 16 weeks of inoculation, no ectomycorrhizas were found. Nevertheless, P. involutusinoculated poplars displayed increased concentrations of soluble sugars and osmolality, leading to an improved water status. Growth was diminished compared with non-inoculated P. euphratica. The presence of hydrogels in the rooting medium resulted in increased biomass and higher plant water content and decreased the osmolality of plant tissues. Drought markedly decreased water contents in rooting medium and plants, and leaf chlorophyll fluorescence, and stimulated the root growth, concentrations of soluble sugars and osmolality in plants. Under drought conditions, P. euphratica exhibited osmoregulation by accumulation of low-molecular-weight carbohydrates.

- These data indicate that adding hydrogels to soils may improve the plant performance. The reasons for improved osmoregulation by fungi and hydrogels were probably related to their stimulating influence on the formation of soluble carbohydrates under drought conditions.
\end{abstract}

Résumé - Le champignon ectomycorhizien Paxillus involutus et les hydrogels influencent les performances de Populus euphratica en condition de stress hydrique.

- Les champignons mycorhiziens et les hydrogels (polymères absorbant l'eau) peuvent améliorer la disponibilité de l'eau pour les arbres. La combinaison des deux facteurs pour améliorer la performance des plants en conditions de limitation d'eau n'a pas encore été étudiée.

- Pour étudier l'influence d'un champignon ectomycorhizien Paxillus involutus, des hydrogels et la combinaison des deux facteurs, un peuplier sensible à la sécheresse, Populus euphratica, a été étudié dans ce travail.

- Après 16 semaines d'inoculation, aucune ectomycorhize n'a été trouvée. Néanmoins, l'inoculation de $P$. involutus aux peupliers a provoqué une augmentation des concentrations de sucres solubles et de l'osmolalité conduisant à une amélioration du statut hydrique. La croissance a été diminuée par rapport à des individus non inoculés. La présence d'hydrogels dans l'enracinement a entraîné une augmentation de la biomasse et une teneur supérieure en eau des plants et une diminution de l'osmolalité des tissus des plants. La sécheresse a diminué sensiblement le contenu en eau des racines, des plants ainsi que la fluorescence de la chlorophylle des feuilles, et stimulé la croissance des racines, les concentrations de sucres solubles et l'osmolalité chez les plants. En condition de sécheresse, $P$. euphratica a présenté une osmorégulation par accumulation d'hydrates de carbone de faible poids moléculaire.

- Ces données indiquent que l'ajout d'hydrogels dans les sols peut améliorer la performance des plants. Les raisons de l'amélioration de l'osmorégulation par les champignons et les hydrogels étaient probablement liées à leur influence sur la stimulation de la formation d'hydrates de carbone solubles en conditions de sécheresse.

\footnotetext{
*Corresponding author: 1zhibin@gwdg.de, luozbbill@163.com
} 


\section{INTRODUCTION}

It has been estimated that currently about $28 \%$ of the earth's land areas are too dry for plant production (IPCC, 2001). It is expected that global warming will increase the severity and frequency of drought (IPCC, 2001; Schär et al., 2004). Drought represents a major constraint on plant growth and productivity (Ciais et al., 2005). For example, the extreme drought and heat wave in 2003 in Europe had significantly negative impacts on tree growth and forest health (Bréda et al., 2006). Furthermore, drought is a major limiting factor for seedling survival, especially during the establishment of forests (Rennenberg et al., 2006). To cope with drought, approaches are required which may alleviate drought stress on seedlings or trees in drought-affected areas and enable establishment of forest plantations. In this respect, mycorrhizal fungi and hydrogels are of interest.

Mycorrhizal fungi can interact with roots of host plants to form mutualistic associations. In this symbiosis, the host plant receives mineral nutrients from the fungus and the fungus obtains photosynthetically-derived carbon compounds from the host (Smith and Read, 1997). Mycorrhizal fungi can improve the water status of plants and increase plant survival and growth under drought conditions (Auge, 2001; Smith and Read, 1997), but the mechanisms of this protection are not clear (Marjanovic et al., 2005; Rincon et al., 2005). For example, in Populus tremuloides seedlings inoculated with ectomycorrhizal fungi, root hydraulic conductance and shoot water potential were higher than in non-mycorrhizal plants (Landhausser et al., 2002). It is generally believed that positive effects of mycorrhizal fungi on host plants are dependent on the formation of mycorrhizas, although there is evidence that mycorrhizal fungi affect host growth and metabolism even if no mycorrhizas are found in inoculated plants (e.g., Dell et al., 1994; Vierheilig et al., 1994).

Hydrogels are polymers that absorb water; sometimes 400 times or more than their own weight, and then release the water progressively along with depletion of soil water. In other words, hydrogels function as an additional water reservoir for the soil-plant-air system (Bhardwaj et al., 2007), and therefore, reduce drought stress on seedlings and trees (Bouranis et al., 1995). Hydrogels may last a few years in the soil before they are degraded into non-toxic components (Holliman et al., 2005). Due to these properties, hydrogels have been used to aid forest establishment and seedling growth in drought-affected areas (Arbona et al., 2005; Hüttermann et al., 1999). The presence of hydrogels in soil prolongs the survival of plants and increases water use efficiency and dry matter production during periods of drought (Arbona et al., 2005; Hüttermann et al., 1999; Viero et al., 2000). Seedlings of Pinus halepensis treated with $0.4 \%(\mathrm{w} / \mathrm{w})$ hydrogels survived drought stress twice as long as those in control soils (Hüttermann et al., 1999). During the drought period, the seedlings treated with hydrogels exhibited three-fold higher growth than those in soil without hydrogels (Hüttermann et al., 1999). Whether hydrogels and mycorrhizas have synergistic effects is unknown.

Populus species are usually grown at riparian sites and most genotypes are drought-sensitive (Sixto et al., 2006). Populus euphratica displays salt tolerance (Gu et al., 2004; Ottow et al., 2005) and grows in arid areas, but recent studies indicate that it may be a drought-sensitive species (Bogeat-Triboulot et al., 2007; Hukin et al., 2005). In the present study, we used this species to investigate whether drought tolerance of $P$. euphratica can be improved by a mycorrhizal fungus (Paxillus involutus) and hydrogels, to find out whether this combination can be used to increase plant performance in drought-affected areas. Plant genotypes have been shown to differ both in their ability to form mycorrhizas, and in the relative benefit received from mycorrhizal colonization (Smith and Read, 1997). Several genotypes of Populus species are able to form ectomycorrhizas with P. involutus (Baum and Makeschin, 2000; Langenfeld-Heyser et al., 2007; Reddy and Satyanarayana, 1998). However, to our knowledge, it is unknown whether $P$. euphratica is able to establish ectomycorrhizas with $P$. involutus. The aims of this study were to investigate (i) whether $P$. euphratica formed ectomycorrhizas with $P$. involutus; and (ii) to find out whether inoculation with $P$. involutus, the presence of hydrogels, or both factors together ameliorated the performance of $P$. euphratica under drought stress. To this end, we determined how the water status in soil and plants, plant growth, soluble sugars, sugar alcohols, and osmolality responded to $P$. involutus, hydrogel and drought treatments in P. euphratica.

\section{MATERIALS AND METHODS}

\subsection{Cultivation of plants and fungi}

Plantlets of $P$. euphratica were multiplied by micropropagation. To acclimatize the plants to ambient conditions, rooted plantlets were cultivated in hydroponic LN-nutrient solutions with low nitrogen supply (modified after Matzner et al., 1982: $300 \mu \mathrm{M} \mathrm{NH} \mathrm{NHO}_{3}$ ) for 3 weeks in a growth room $\left(21^{\circ} \mathrm{C}, 50-60 \%\right.$ relative air humidity, $16 \mathrm{~h}$ of light per day at $150 \mu \mathrm{mol}$ photons $\mathrm{m}^{-2} \mathrm{~s}^{-1}$ ) before transfer to a climate chamber with conditions similar to those in the growth room.

Paxillus involutus (Bartsch.) (Strain MAJ in the Göttingen stock collection, initially collected in France under a poplar tree) was grown on 2\% Modified Melin-Norkrans agar medium (MMN) and subsequently in liquid culture medium as described previously (Langenfeld-Heyser et al., 2007).

\subsection{Inoculation, rooting medium and hydrogels}

Mycelia in liquid culture were used for inoculation. The upper clear supernatant of the culture medium was discarded, and $50 \mathrm{~mL}$ mycelia of $P$. involutus were homogenized and used to inoculate the rooting medium. The rooting medium consisted of 5 parts peat, 5 parts fine sand (grain size: $0.1-0.3 \mathrm{~mm}$ ) and 10 parts coarse sand (grain size: $1.2-2.0 \mathrm{~mm}$ ). The rooting medium was sterilized in Erlenmeyer flasks (120 ${ }^{\circ} \mathrm{C}, 20 \mathrm{~min}$, Zirbus HST 666). Subsequently, it was inoculated with mycelia of $P$. involutus or only with liquid culture medium without mycelia ( $50 \mathrm{~mL}$ for $100 \mathrm{~mL}$ of rooting medium) and incubated for 3 weeks in an acclimatized dark room $\left(22^{\circ} \mathrm{C}, 67 \%\right.$ relative air humidity) before transfer to the growth tubes.

The rest of the rooting medium was also sterilized. Half of the rooting medium was mixed with hydrogels (Stockosorb K 400, 
Chemische Fabrik Stockhausen GmbH Co. KG, Krefeld, Germany) at a concentration of $0.6 \%(\mathrm{w} / \mathrm{w})$. Growth tubes with a nylon mesh at the bottom were filled with ca. $500 \mathrm{~mL}$ rooting medium with or without hydrogels $[0.6 \%(\mathrm{w} / \mathrm{w})]$ and $100 \mathrm{~mL}$ rooting medium inoculated or not inoculated with $P$. involutus, yielding the following four treatments: (i) control, (ii) hydrogels, (iii) fungus and (iv) hydrogels and fungus. Subsequently, poplar plantlets were planted in the growth tubes. The plants were randomized twice a week. Each plant was irrigated daily with $20 \mathrm{~mL}$ sterilized LN-nutrient solution in the morning and $20 \mathrm{~mL}$ sterilized water in the evening. Poplar plants were maintained in the climate chamber for 16 weeks before drought stress.

\subsection{Drought stress and harvest}

The watered plants were irrigated to field capacity with sterilized water twice a day. Half of the plants of each of the four treatments were exposed to water deficit by stopping irrigation. After 5 days of drought stress, the watered and stressed plants were harvested. The plants were separated into leaves, stem and roots for fresh mass determination. Stem heights and length of main roots were also measured. About $100 \mathrm{~g}$ of the rooting medium was collected for gravimetrical determination of water contents.

For biochemical analysis, samples of fresh leaves, stem and roots were immediately frozen in liquid nitrogen and subsequently stored at $-80{ }^{\circ} \mathrm{C}$. Samples were milled into fine powder with a ball mill (Retsch, Haan, Germany), pre-cooled in liquid nitrogen. Aliquots of fine powder were used for determination of the ratio of fresh to dry mass and the relative water contents (RWC) in plants after $48 \mathrm{~h}$ of drying at $60{ }^{\circ} \mathrm{C}$. RWC was calculated as follows: $\mathrm{RWC} \%=($ Fresh weight - Dry weight)/Fresh weight $\times 100$.

\subsection{Leaf chlorophyll fluorescence yield measurements}

The chlorophyll fluorescence of trees was measured regularly under ambient light with a photosynthesis yield analyzer (Mini-PAM, Walz, Effeltrich, Germany) in the chamber and calculated as quantum yield of PSII $(\Phi)$ :

$\Phi=\left(F_{\mathrm{m}}-F_{0}\right) / F_{\mathrm{m}}$ (Kitajima and Butler, 1975), where $F_{\mathrm{m}}$ and $F_{0}$ are the maximum and basic fluorescence levels, respectively.

\subsection{Formation of mycorrhizas}

Root systems of $P$. euphratica inoculated or not inoculated with $P$. involutus strain MAJ were spread in Petri dishes and the whole plant root system was examined under a binocular for ectomycorrhizal root tips.

\subsection{Determination of sugars and sugar alcohols}

Soluble sugars (glucose, fructose and sucrose) and starch in leaves and stems were determined as described previously (Luo et al., 2006).

Carbohydrates in roots were analyzed in detail since they play major roles in osmotic adjustment. About $50 \mathrm{mg}$ of freeze-dried materials for each sample were extracted and analyzed by GC/MS as described by Hu et al. (2005).

\subsection{Determination of osmotic pressure}

The osmolality of leaves, stems and roots was determined by freezing point depression with an osmometer (Knauer, Halbmikro ML Osmometer, Germany) according to the instructions of the manufacturer.

\subsection{Statistical analysis}

All statistical tests were performed with Statgraphics (STN, St. Louis, MO, USA). When interactions were significant, an a posteriori comparison of means was made. To reduce the chance of type I errors, all $P$-values of these multi-comparisons were corrected by the TukeyHSD method. Data were tested for normality with the Shapiro-Wilks test. Differences between parameter means were considered significant when the $P$-value of the ANOVA $F$-test was less than 0.05 .

\section{RESULTS}

\subsection{Formation of ectomycorrhizas}

Ectomycorrhizas were not found in root systems of $P$. euphratica inoculated with $P$. involutus after growing inoculated plants for 16 weeks in a climate chamber. The plants grown in soil without inoculation were also examined and no ectomycorrhizas were detected.

\subsection{Water contents in soil and plants}

Soil water contents (SWC) and the relative water contents (RWC) of plants were determined at harvest (Tab. I). SWC was unaltered in rooting medium inoculated with $P$. involutus compared with the soil mixture without inoculation. As expected, SWC was significantly higher $(+36 \%)$ in rooting medium with hydrogels than in medium without hydrogels. SWC markedly decreased (-80\%) under drought conditions in comparison with well-watered conditions.

RWC in roots, stems and leaves were 65.6-83.6\%, $52.2-64.1 \%$ and $50.1-79.4 \%$, respectively, depending on the treatments. RWC in roots and stems significantly increased by 5 and $9 \%$, respectively, in inoculated in comparison with non-inoculated plants. RWC in roots, stems and leaves also increased 7, 4 and $11 \%$, respectively, in plants with hydrogels compared with plants without hydrogel addition. RWC in roots, stems and leaves markedly decreased by 11,8 and $15 \%$, respectively, in plants exposed to drought compared with wellwatered plants.

\subsection{Biometric measurements}

Stem height, length of main roots and leaf biomass of plants remained unaffected by inoculation with $P$. involutus (Figs. 1 
Table I. The gravimetric soil water content and the RWC in roots, stem and leaves at the end of the drought stress of $P$. euphratica grown in a climate chamber. Data indicates mean $\pm \mathrm{SE}(n=6)$. The plantlets of $P$. euphratica were either inoculated with $P$. involutus $(\mathrm{P})$ or not inoculated with this fungus, with either added hydrogels $(\mathrm{H})$ or no hydrogels in the soil and either drought-stressed (D) or normally watered (W). Significance levels are shown for the $P$-values of the ANOVAs of $P$. involutus strain MAJ - Paxin, Hydrogel and Drought, $* P<0.05$; ** $P<0.01$; *** $P<0.001$; **** $P<0.0001$. No interactions were significant.

\begin{tabular}{lcccc}
\hline Treatments & Soil water content $(\%)$ & RWC in roots $(\%)$ & RWC in stem $(\%)$ & RWC in leaves $(\%)$ \\
\hline D & $1.27 \pm 0.02$ & $65.6 \pm 1.1$ & $52.2 \pm 1.1$ & $63.09 \pm 0.41$ \\
W & $10.67 \pm 0.03$ & $77.6 \pm 1.2$ & $58.2 \pm 2.4$ & $73.62 \pm 0.46$ \\
HD & $2.77 \pm 0.02$ & $74.7 \pm 1.8$ & $55.2 \pm 0.9$ & $70.79 \pm 0.46$ \\
HW & $12.93 \pm 0.02$ & $82.1 \pm 1.1$ & $59.6 \pm 0.9$ & $74.13 \pm 0.46$ \\
PD & $1.23 \pm 0.01$ & $68.5 \pm 1.3$ & $56.7 \pm 0.9$ & $50.12 \pm 0.47$ \\
PW & $7.77 \pm 0.02$ & $83.6 \pm 1.2$ & $64.1 \pm 1.2$ & $79.43 \pm 0.41$ \\
PHD & $3.73 \pm 0.02$ & $79.9 \pm 1.2$ & $62.8 \pm 1.3$ & $75.86 \pm 0.45$ \\
PHW & $13.20 \pm 0.03$ & $82.2 \pm 0.9$ & $63.9 \pm 1.0$ & $79.43 \pm 0.41$ \\
\hline Paxin & $* *$ & $* * *$ & $* *$ & $* * *$ \\
Hydrogel & $* * * * * * * *$ & $* * *$ & $* *$ \\
Drought & & $* * *$ & & $*$ \\
\hline
\end{tabular}

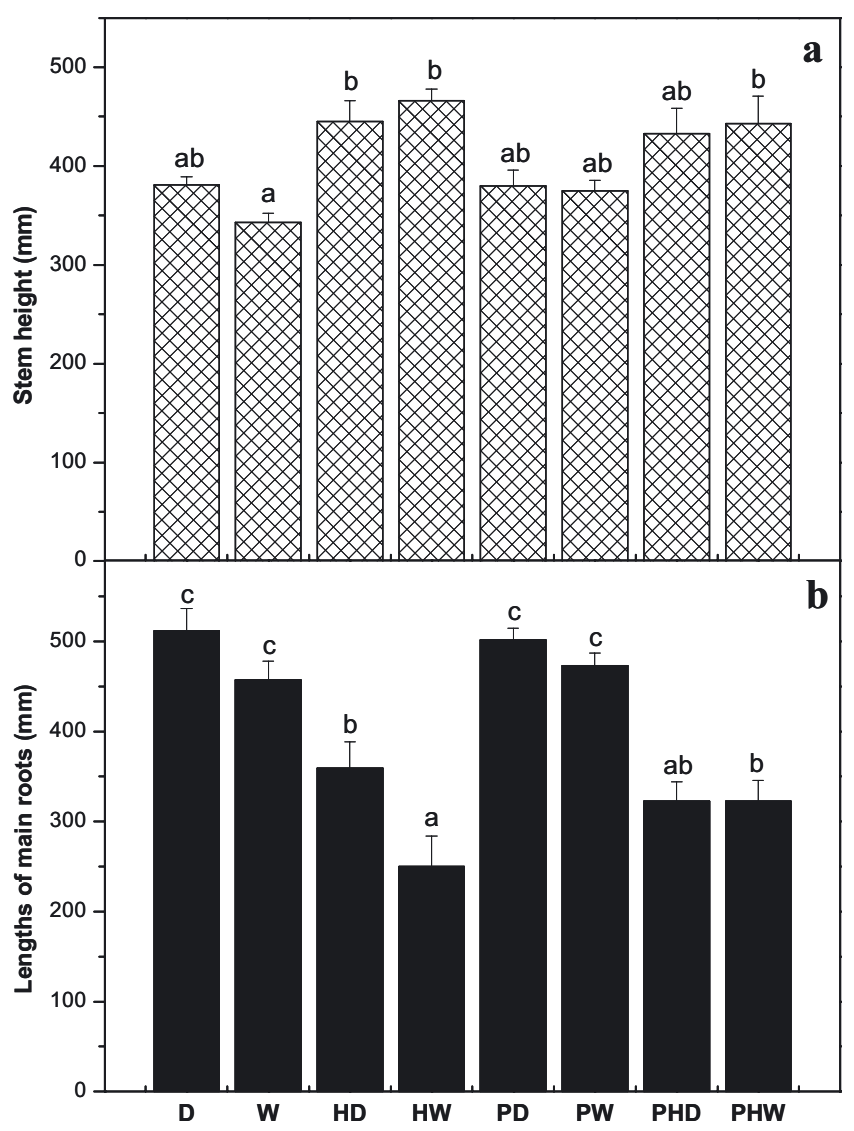

Figure 1. Stem height (a) and lengths of main roots (b) of P. euphratica. The plantlets of $P$. euphratica were either inoculated with $P$. involutus $(\mathrm{P})$ or not inoculated with this fungus, with either added hydrogels $(\mathrm{H})$ or no hydrogels in the soil and either drought-stressed (D) or normally watered $(\mathrm{W})$. The bar indicates mean $\pm \mathrm{SE}(n=6)$. Different letters for the same parameter indicate significant difference.

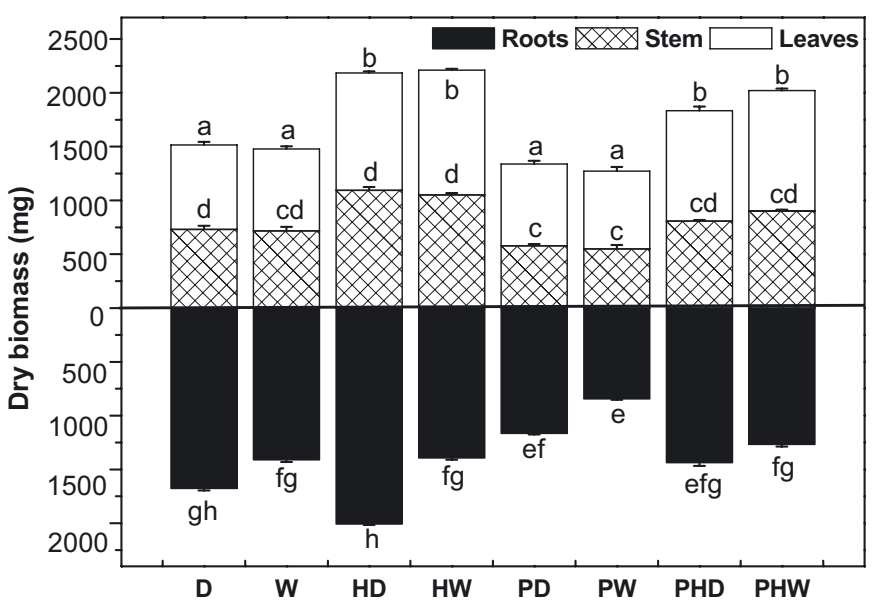

Figure 2. Dry biomass of roots, stem and leaves of $P$. euphratica. The plantlets of $P$. euphratica were either inoculated with $P$. involutus $(\mathrm{P})$ or not inoculated with this fungus, with either added hydrogels $(\mathrm{H})$ or no hydrogels in the soil and either drought-stressed (D) or normally watered $(\mathrm{W})$. The bar indicates mean $\pm \mathrm{SE}(n=6)$. Different letters for the same parameter indicate significant difference.

and 2, Table II). Interestingly, plants inoculated with the fungus had less root $(-27 \%)$ and stem $(-21 \%)$ biomass than those grown without inoculum. Plants grown with hydrogels had significantly higher stem height $(+17 \%)$, and biomass of roots $(+17 \%)$, stems $(+33 \%)$ and leaves $(+31 \%)$ than those grown without hydrogels. However, the length of the main root was shorter $(-35 \%)$ in plants grown in the presence compared with those grown in the absence of hydrogels. Stem height and biomass of leaves and stems were unaffected by drought stress. 
Table II. Statistical results of aboveground height, length of main root, and dry weight (DW) of roots, stem and leaves of $P$. euphratica grown in a climate chamber. The plantlets of $P$. euphratica were either inoculated with $P$. involutus (strain MAJ) or not inoculated with this fungus, with either added hydrogels or no hydrogels in the soil and either drought-stressed or normally watered. Significance levels are shown for the $P$-values of the ANOVAs of $P$. involutus strain MAJ Paxin, Hydrogel and Drought, $* P<0.05$; ** $P<0.01$; *** $P<$ 0.001 ; **** $P<0.0001$. No interactions were significant.

\begin{tabular}{lccccc}
\hline Source & Height & $\begin{array}{r}\text { Length of } \\
\text { main root }\end{array}$ & $\begin{array}{c}\text { DW of } \\
\text { roots }\end{array}$ & $\begin{array}{c}\text { DW of } \\
\text { leaves }\end{array}$ & $\begin{array}{c}\text { DW of } \\
\text { stem }\end{array}$ \\
\hline Paxin & & & $* * *$ & & $*$ \\
Hydrogel & $* *$ & $* * * *$ & $*$ & $* * * *$ & $*$ \\
Drought & & $* * *$ & $*$ & & \\
\hline
\end{tabular}

The length of main roots and biomass of roots were increased 11 and $22 \%$, respectively, by drought stress.

\subsection{Yield of chlorophyll fluorescence}

The quantum yield of PSII $(\Phi)$ was measured regularly under light (Fig. 3). It was noted that $\Phi$ was significantly lower in plants grown in soils inoculated with $P$. involutus than in those without inoculation. $\Phi$ was higher in plants with hydrogels than in plants without hydrogel addition. $\Phi$ was significantly decreased in plants under drought conditions in comparison with well-irrigated plants.

\subsection{Carbohydrates}

Concentrations of soluble carbohydrates (glucose + fructose + sucrose) and starch in stems and leaves are shown in Figure 4 and Table III. Leaves of plants inoculated with $P$. involutus contained significantly higher concentrations of sucrose and total soluble sugar concentrations, but decreased concentrations of fructose, than non-inoculated plants. Plants with hydrogels contained increased concentrations of starch and soluble sugars, but decreased concentrations of glucose in leaves.

In their stems, plants inoculated with $P$. involutus contained significantly elevated concentrations of glucose, fructose and sucrose compared with non-inoculated plants. Plants with hydrogels contained decreased concentrations of sugars in their stems compared with plants without hydrogels.

In response to drought, concentrations of sugars in leaves and stems were markedly increased, which is apparently mainly due to the hydrolysis of starch under drought conditions compared with watered conditions.

In roots, concentrations of carbohydrates were analyzed in detail and glucose, fructose, sucrose, inositol, galactose, mannitol, sorbitol and trehalose were found (Fig. 5 and Tab. IV). Glucose, fructose and sucrose accounted for more than $95 \%$ of total sugars analyzed and hence were the major soluble sugars in roots (Fig. 5a). Although the other compounds also showed

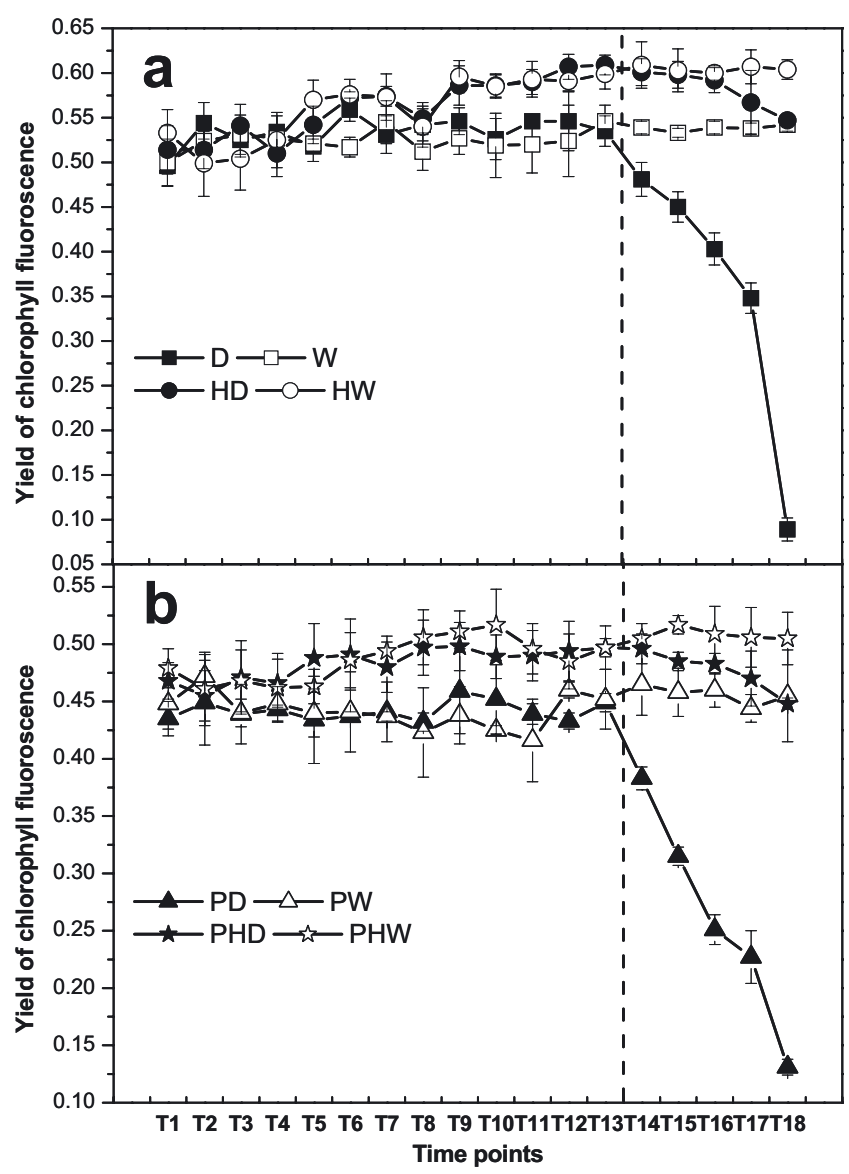

Figure 3. Yield of chlorophyll fluorescence in leaves of P. euphratica. The plants were cultivated in a growth chamber and were either not inoculated with $P$. involutus in Figure 3 a or inoculated with $P$. involutus in Figure $3 \mathrm{~b}$ and grown without or with added hydrogels $(\mathrm{H})$ in the soil for 16 weeks. Plants were either normally watered (W) or drought-stressed (D) for 5 days at the end of the experiment. The dashed line indicates the beginning of the drought stress. The symbol indicates mean $\pm \operatorname{SE}(n=6)$.

Table III. Statistical results of carbohydrates in leaves and stem of $P$. euphratica grown in a climate chamber. The plantlets of $P$. euphratica were either inoculated with $P$. involutus (strain MAJ) or not inoculated with this fungus, with either added hydrogels or no hydrogels in the soil and either drought-stressed or normally watered. Significance levels are shown for the $P$-values of the ANOVAs of $P$. involutus strain MAJ - Paxin, Hydrogel and Drought, $* P<0.05$; ** $P<0.01$; *** $P<0.001$; **** $P<0.0001$. No interactions were significant.

\begin{tabular}{ccccccc}
\hline Tissue & Source & Glucose & Fructose & Sucrose & Starch & Sum \\
\hline \multirow{2}{*}{ Leaves } & Paxin & & $*$ & $* *$ & & $*$ \\
& Hydrogel & $* * * *$ & & & $* * * *$ & $* * * *$ \\
& Drought & $* * * *$ & $* * * *$ & $*$ & $*$ & \\
Stem & Paxin & $* * * *$ & $* * * *$ & $* *$ & & \\
& Hydrogel & $* * * *$ & $* * * *$ & & & $*$ \\
& Drought & $* * * *$ & $* * * *$ & $* *$ & $* * *$ & \\
\hline
\end{tabular}




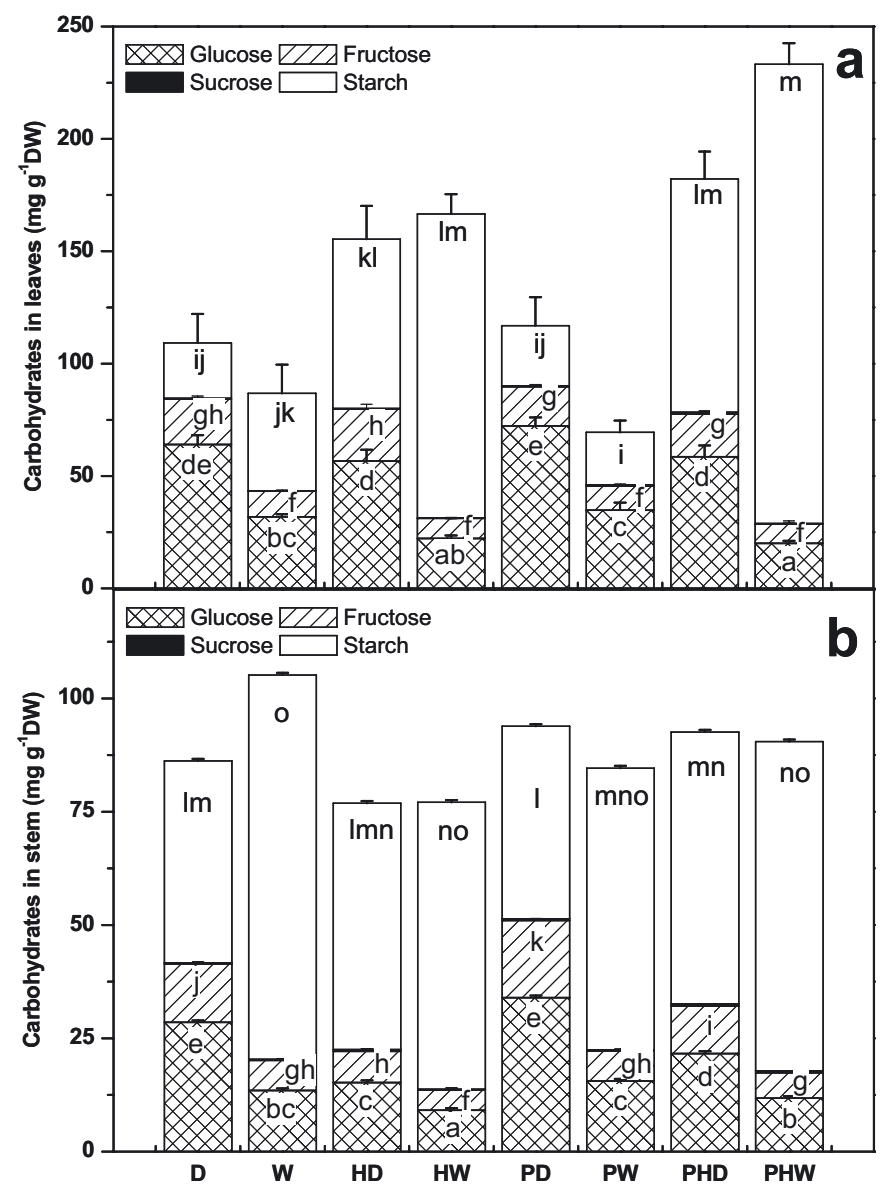

Figure 4. Carbohydrates in leaves (a) and stem (b) of P. euphratica. The plantlets of $P$. euphratica were either inoculated with $P$. involutus $(\mathrm{P})$ or not inoculated with this fungus, with either added hydrogels $(\mathrm{H})$ or no hydrogels in the soil and either drought-stressed (D) or normally watered $(\mathrm{W})$. The bar indicates mean $\pm \mathrm{SE}(n=6)$. Different letters for the same parameter indicate significant difference.

significant fluctuations with treatments, they contributed only marginally to osmotic adjustment. Therefore, the following description will focus only on the major soluble sugars.

For the fungus treatment, under well-watered conditions, concentrations of sucrose, glucose and fructose in roots grown in soil inoculated with $P$. involutus were markedly higher than those in roots in soil with non-inoculation (Fig. 5a, PW-W). When hydrogels were added to the substrate, the concentration of sucrose in roots grown in soil inoculated with $P$. involutus was significantly higher than that in roots grown in soil with non-inoculation (Fig. 5a, PHW-HW). Under drought conditions, sucrose content in roots in soil inoculated with $P$. involutus was markedly higher than that in roots in soil with non-inoculation (Fig. 5a, PD-D). When hydrogels were added to the substrate, levels of sucrose and glucose in roots in soil inoculated with $P$. involutus were significantly elevated

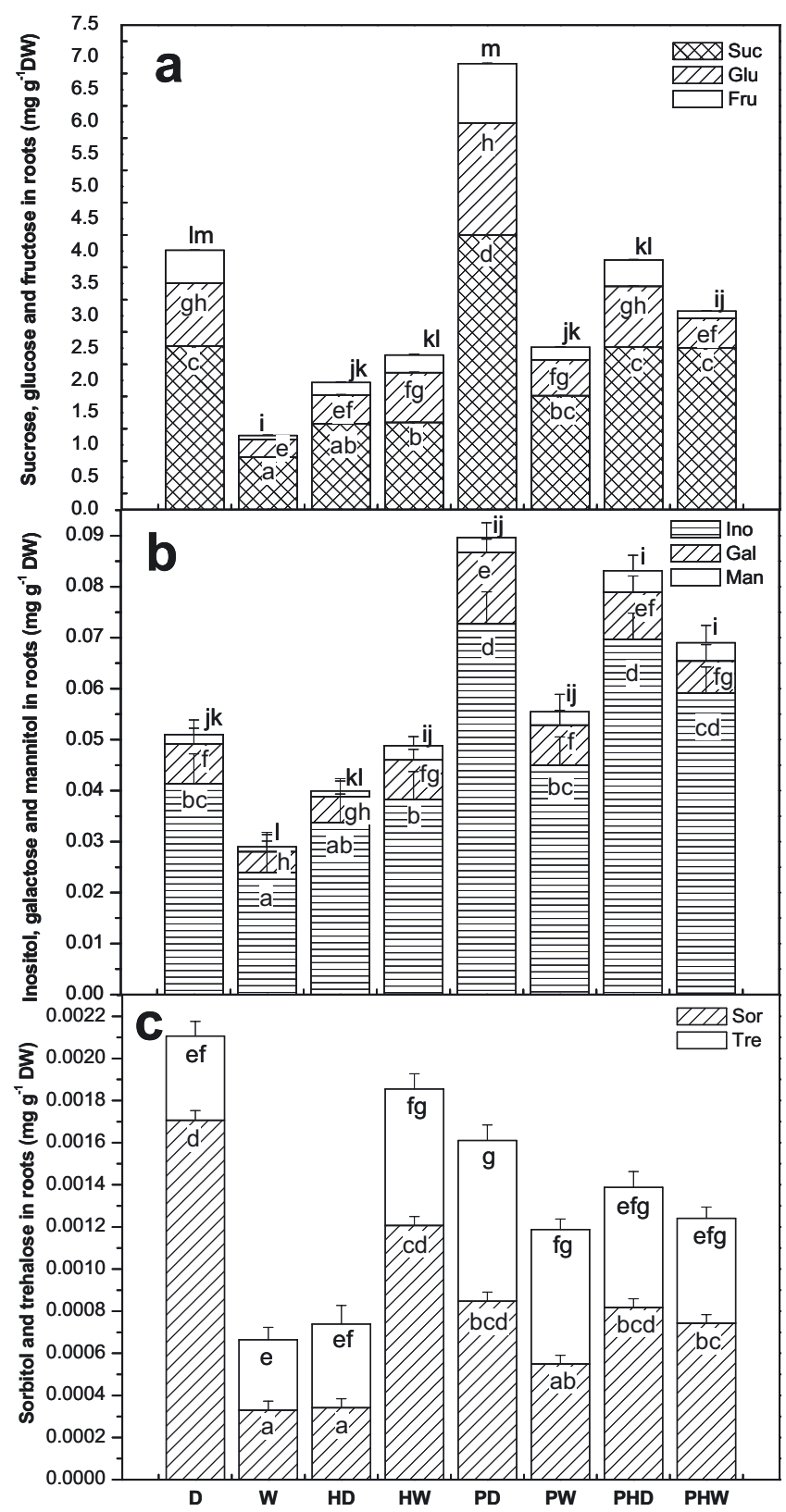

Figure 5. Sugars and sugar alcohols in roots of $P$. euphratica. The plantlets of $P$. euphratica were either inoculated with $P$. involutus $(\mathrm{P})$ or not inoculated with this fungus, with either added hydrogels $(\mathrm{H})$ or no hydrogels in the soil and either drought-stressed (D) or normally watered $(\mathrm{W})$. The bar indicates mean $\pm \mathrm{SE}(n=6)$. Different letters for the same parameter indicate significant difference.

in comparison with those in roots in soil with non-inoculation (Fig. 5a, PHD-HD).

For hydrogel treatment, under well-watered conditions, contents of sucrose, glucose and fructose in roots in soil with hydrogel addition were pronouncedly higher than those in roots in soil without hydrogel addition (Fig. 5a, HW-W), but these effects disappeared when the soil was inoculated with P. involutus (Fig. 5a, PHW-PW). Under drought conditions, 
Table IV. Statistical results of sugars and sugar alcohols in roots of $P$. euphratica grown in a climate chamber. The plantlets of $P$. euphratica were either inoculated with $P$. involutus (strain MAJ) or not inoculated with this fungus, with either added hydrogels or no hydrogels in the soil and either drought-stressed or normally watered. Significance levels are shown for the $P$-values of the ANOVAs of $P$. involutus strain MAJ Paxin, Hydrogel and Drought, $* P<0.05$; ** $P<0.01$; *** $P<0.001$; **** $P<0.0001$. No interactions were significant.

\begin{tabular}{|c|c|c|c|c|c|c|c|c|c|}
\hline Source & Glucose & Fructose & Sucrose & Inositol & Galactose & Mannitol & Sorbitol & Trehalose & Sum \\
\hline Paxin & $*$ & & $* * * *$ & $* * * *$ & $* * *$ & $* * * *$ & & $*$ & $* * * *$ \\
\hline Hydrogel & & & & & & $*$ & & & \\
\hline Drought & $* * *$ & $* * * *$ & $* * *$ & $* *$ & $* *$ & & & & $* * * *$ \\
\hline
\end{tabular}

levels of sucrose, glucose and fructose in roots in soil with hydrogel addition were markedly lower than those in roots in soil without hydrogel addition (Fig. 5a, HD-D). When soil was inoculated with $P$. involutus, levels of sucrose and fructose were significantly lower in roots in soil with hydrogel addition than those in roots in soil without hydrogel addition (Fig. 5a, PHD-PD).

Drought markedly increased contents of sucrose, glucose and fructose in roots compared with those in well-watered roots (Fig. 5a, D-W), but these effects disappeared when hydrogels were added to soil (Fig. 5a, HD-HW). When soil was inoculated with $P$. involutus, levels of these sugars in roots exposed to drought were also markedly elevated compared with those in well-watered roots (Fig. 5a, PD-PW). When hydrogels and $P$. involutus were both added to soil, levels of glucose and fructose in roots were markedly enhanced in comparison with those in well-watered roots (Fig. 5a, PHD-PHW).

\subsection{Osmolality}

Osmolalities determined in different poplar tissues increased in the order roots $<$ stem $<$ leaves (Fig. 6 and Tab. V). Since changes in osmolality in roots, stem and leaves had a similar pattern (Fig. 6), the following description will focus only on osmolality changes in leaves (Fig. 6a).

For the fungus treatment, under well-watered conditions, osmolality in leaves of $P$. euphratica grown in soil inoculated with $P$. involutus remained unchanged in comparison with that in soil with non-inoculation of this fungus, irrespective of hydrogel addition (Fig. 6a, PW-W and PHW-HW). Under drought conditions, osmolality in leaves of $P$. euphratica grown in soil inoculated with $P$. involutus was markedly higher than that in soil with non-inoculation (Fig. 6a, PD-D), but this effect disappeared when hydrogels were added to soil (Fig. 6a, PHD-HD).

For hydrogel treatment, under well-watered conditions, osmolality in leaves of $P$. euphratica grown in soil with hydrogel addition remained unaltered compared with that in soil without hydrogel addition, irrespective of inoculation with $P$. involutus (Fig. 6a, HW-W and PHW-PW). Under drought conditions, osmolality in leaves of $P$. euphratica grown in soil with hydrogel addition was significantly lower than that in soil without hydrogel addition, irrespective of inoculation with $P$. involutus (Fig. 6a, HD-D and PHD-PD).

For drought treatment, osmolality in leaves of $P$. euphratica exposed to drought stress was significantly higher than that in well-watered leaves, irrespective of hydrogel addition and/or inoculation with $P$. involutus (Fig. 6a, D-W, HD-HW, PD-PW and PHD-PHW).

To investigate whether measured osmolality was correlated with osmolality produced by the major soluble sugars (glucose + fructose + sucrose), regression analysis was conducted (Fig. 7). These data indicate that at the tissue as well as at whole-plant level, positive correlations were observed between total osmolality and osmolality from soluble sugars.

\section{DISCUSSION}

\subsection{Non-host responses of $P$. euphratica to inoculation with $P$. involutus}

The present study indicates that $P$. euphratica is unable to form mycorrhizas with $P$. involutus, although many genotypes of Populus species are host trees for this ectomycorrhizal species (Baum and Makeschin, 2000; LangenfeldHeyser et al., 2007; Reddy and Satyanarayana, 1998). To exclude possible limitations of our experimental system, we also tested an axenic Petri dish system which is highly effective for mycorrhiza formation, as shown by Hampp et al. (1996), and which functioned very well with Paxillus involutus (strain MAJ) and P. x canescens (Gafur et al. 2004). Again, no ectomycorrhizas were found in fine roots of $P$. euphratica inoculated with $P$. involutus (data not shown). These observations underline that $P$. euphratica is not a competent host species for $P$. involutus.

Although ectomycorrhizas with $P$. involutus were not formed, an effect of fungal inoculation on plant growth and metabolism was observed. This might have been due to interactions, which can occur between fungi and plants, even if no symbiosis is built up (e.g., Dell et al., 1994; Vierheilig et al., 1994). P. euphratica benefited from the fungus by improved relative water contents, increased concentrations of sucrose and glucose in roots and elevated osmolality in leaves. Negative effects of the fungus on plants were decreases in growth of roots and stem and reduced yield of chlorophyll fluorescence in leaves. It is possible that the improved water status of plants inoculated with the fungus were due to modifications in soil structures or facilitated capillary water transport along the hyphal cell walls. The fungal partner in the ectomycorrhizal association is able to attract sugars towards the root system, leading to higher concentrations of carbohydrates than 


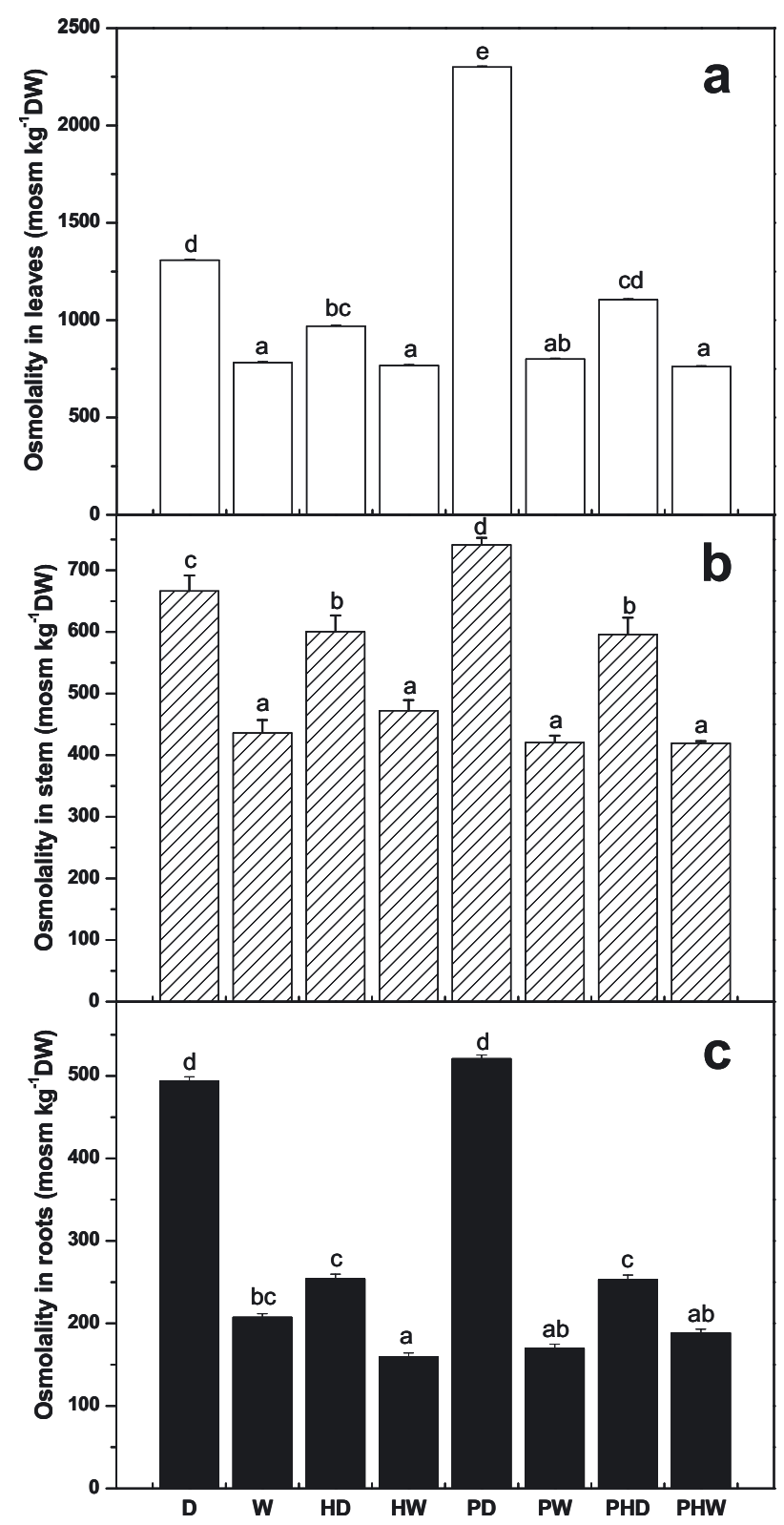

Figure 6. Osmolality in leaves (a), stem (b) and roots (c) of $P$. euphratica. The plantlets of $P$. euphratica were either inoculated with $P$. involutus $(\mathrm{P})$ or not inoculated with this fungus, with either added hydrogels $(\mathrm{H})$ or no hydrogels in the soil and either drought-stressed (D) or normally watered (W). The bar indicates mean $\pm \mathrm{SE}(n=6)$. Different letters for the same parameter indicate significant difference.

in non-mycorrhizal roots (Hampp and Schaeffer, 1999; Nehls et al., 2001; Smith and Read, 1997). However, in our study the enhanced soluble sugar concentrations in roots of plants inoculated with $P$. involutus were not a consequence of ectomycorrhiza formation. It is possible that the fungus affects sink capacities by hormonal effects (Nehls et al., 2007). Indirect effects must also be considered. The increased sugar contents in roots might be due to the water competition with fun-
Table V. Statistical results of osmolality in roots, stem and leaves of $P$. euphratica grown in a climate chamber. The plantlets of $P$. euphratica were either inoculated with $P$. involutus (strain MAJ) or not inoculated with this fungus, with either added hydrogels or no hydrogels in the soil and either drought-stressed or normally watered. Significance levels are shown for the $P$-values of the ANOVAs of $P$. involutus strain MAJ - Paxin, Hydrogel and Drought, $* P<0.05$; ** $P<0.01$; *** $P<0.001$; **** $P<0.0001$. No interactions were significant.

\begin{tabular}{lccc}
\hline Source & Leaf & Stem & Root \\
\hline Paxin & $* *$ & & \\
Hydrogel & $* * * *$ & $* *$ & $* * * *$ \\
Drought & $* * * *$ & $* * * *$ & $* * * *$ \\
\hline
\end{tabular}

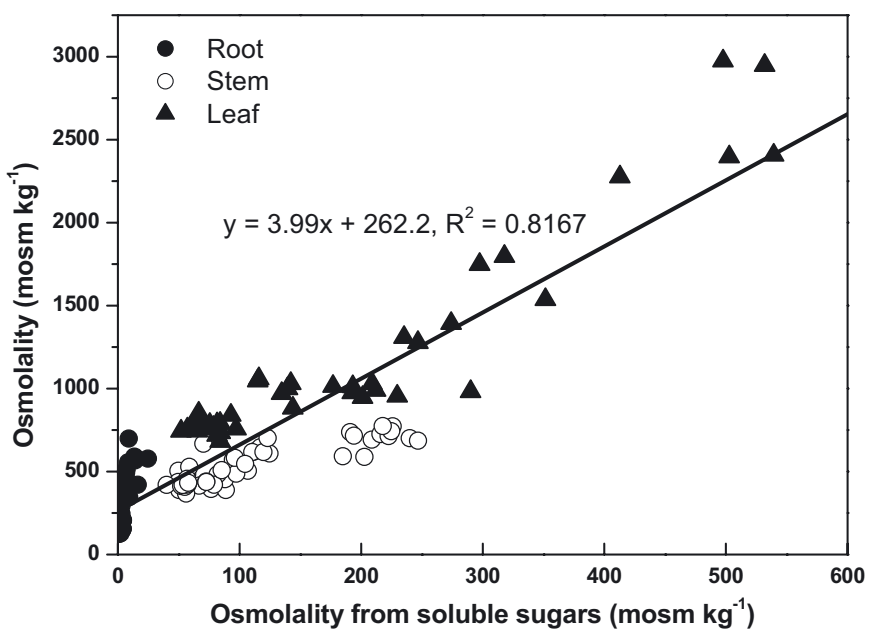

Figure 7. The correlation between osmolality of soluble sugars (glucose + fructose + sucrose) and total osmolality in roots (closed circles), stem (open circles) and leaves (closed triangles) of P. euphratica grown in a climate chamber. The correlation was statistically significant at $P<0.0001$.

gal hyphae. The accumulation of sucrose and glucose in roots of poplar inoculated with $P$. involutus might be required for successful competition by keeping roots at higher osmotic potentials. The negative effects of the fungus on the plant could also be the result of this competition if the carbon costs of this acclimation were high.

It has been documented that mycorrhiza formation can improve water availability for the host plants due to the large contact area between fungal mycelia and soil and the enhanced water-transporting capacity (Marjanovic et al., 2005). It is believed that the benefit the host plant gains with respect to drought tolerance is based on mycorrhiza formation. However, here we report that the improved water status in the plants can also occur even if no mycorrhizas were formed between the plant and the fungus. 


\subsection{Influence of hydrogels on $P$. euphratica}

Hydrogels applied to the rooting medium may absorb water during irrigation and then release the water progressively along with the water depletion in the medium. This feature of hydrogels is critical for improving plant growth in soils or media with low water-holding capacity. Due to the water reservoir provided by hydrogels in the rooting medium, soil water content, plant water status and photosynthesis, as indicated by chlorophyll fluorescence of leaves, were enhanced. Increased plant height growth and biomass were probably also a consequence of the improved water status. These findings suggest that assimilated carbon is more efficiently utilized to build biomass in plants with hydrogels compared with plants without hydrogels. Effective conversion of assimilated carbon may have led to decreases in soluble sugar concentrations in plants grown in the presence of hydrogels, and thus a drop in osmolality. Another explanation is that in soil with hydrogels, higher water-holding capacity does not required decreases in the osmotic potential to drive plant water uptake. Thus, soluble carbohydrates can be inverted into starch or biomass. Overall, our data are consistent with previous studies, which have demonstrated that application of hydrogels to soils improved plant performance (Arbona et al., 2005; Viero et al., 2006). For instance, Arbona et al. (2005) found that application of hydrogels to the substrate increased water content, leaf water potential, root biomass, $\mathrm{CO}_{2}$ assimilation and stomatal conductance of citrus trees.

It is interesting that the root biomass increased but the lengths of main roots decreased due to the application of hydrogels in rooting medium (Figs. 1b and 2). This was caused by changes in root morphology, which were also observed during the examination of the roots under a stereomicroscope (data not shown). The main roots in plants with hydrogels were thicker (bigger diameter) and the root tips were blunt, which is quite different from the sharp root tips of plants without hydrogels. These morphological changes in main roots may be an adjustment to the improved water status of plants with hydrogels.

\subsection{Influence of drought on P. euphratica}

Recently, responses of $P$. euphratica to gradual soil water deficit have been studied in detail at the protein, transcript and metabolite levels, but most of the analyses focused on leaves (Bogeat-Triboulot et al., 2007). Information on the physiological changes in drought-stressed $P$. euphratica roots may be especially relevant for practical applications, e.g. when growing this species in drought-affected areas. In this study, $P$. euphratica exposed to drought stress exhibited decreases in water status and leaf chlorophyll fluorescence, and increases in the length of main roots, root biomass, concentrations of soluble sugars and osmolality, which are common responses of plants to water deficit (Polle et al., 2006). When the tissue water content was not sufficient to maintain normal physiological functions, $P$. euphratica had to reduce the water consumption by stomatal closure. This may have led to decreases in chlorophyll fluorescence, reflecting decreases in photosynthetic electron transport.

Accumulation of low-molecular-weight carbohydrates is an effective strategy for $P$. euphratica to cope with drought stress. The sum of glucose, fructose and sucrose, i.e. the major soluble carbohydrates in roots, stem, and leaves of P. euphratica were 84, 99 and 123\% increased compared with the irrigated plants. Since glucose, fructose and sucrose are major osmolytes in trees exposed to drought conditions (Merchant et al., 2006), the accumulation of these sugars and the positive correlation with osmolality indicate that these carbohydrates play an essential role in osmoregulation in P. euphratica. Since the slope of the positive correlation line was higher than 1 (Fig. 7), this suggests that other osmolytes (e.g. ions, sugar alcohols) may also be important contributors to the osmotic pressure in $P$. euphratica.

Taken together, $P$. euphratica displayed an improved water status in roots and stem due to the presence of $P$. involutus in the rooting medium, although no mycorrhizas were formed. Plant biomass decreased in response to the inoculation. P. euphratica grown in the rooting medium with hydrogels displayed an improved water status and exhibited enhanced growth. However, no synergistic effects on plant performance were found in the presence of hydrogels in combination with fungal inoculation, suggesting that both factors influence plant metabolism independently.

Acknowledgements: We are grateful to the Deutsche Forschungsgemeinschaft for providing financial support to the PRG (Poplar Research Group, Germany) for Andrea Polle and to the Deutscher Akademischer Austauschdienst (DAAD) for funding a postdoctoral scholarship for Zhi-Bin Luo in the program "Modern Application of Biotechnology".

\section{REFERENCES}

Arbona V., Iglesias D.J., Jacas J., Primo-Millo E., Talon M., and GomezCadenas A., 2005. Hydrogel substrate amendment alleviates drought effects on young citrus plants. Plant Soil 270: 73-82.

Auge R.M., 2001. Water relations, drought and vesicular-arbuscular mycorrhizal symbiosis. Mycorrhiza 11: 3-42.

Baum C. and Makeschin F., 2000. Effects of nitrogen and phosphorus fertilization on mycorrhizal formation of two poplar clones (Populus trichocarpa and P. tremula $\times$ tremuloides). J. Plant Nutr. Soil Sci. 163: 491-497.

Bhardwaj A.K., Shainberg I., Goldstein D., Warrington DN., and Levy GJ., 2007. Water retention and hydraulic conductivity of cross-linked polyacrylamides in sandy soils. Soil Sci. Soc. Am. J. 71: 406-412.

Bogeat-Triboulot M., Brosche M., Renaut J., Jouve L., Thiec D.L., Fayyaz P., Vinocur B., Witters E., Laukens K., Teichmann T., Altman A., Hausman J., Polle A., Kangasjärvi J., and Dreyer E., 2007. Gradual soil water depletion results in reversible changes of gene expression, protein profiles, ecophysiology, and growth performance in Populus euphratica, a poplar growing in arid regions. Plant Physiol. 143: 876-892.

Bréda N., Huc R., Granier A., and Dreyer E., 2006. Temperate forest trees and stands under severe drought: a review of ecophysiological responses, adaptation processes and long-term consequences. Ann. For. Sci. 63: 625-644. 
Bouranis D.L., Theodoropoulos A.G., and Drossopoulos J.B., 1995. Designing synthetic polymers as soil conditioners. Commun. Soil Sci. Plant Anal. 26: 1455-1480.

Ciais P.H., Reichstein M., Viovy N., Granier A., Allard V. et al., 2005. Europe-wide reduction in primary productivity caused by the heat and drought in 2003. Nature 437: 529-533.

Dell B., Malajczuk N., Bougher N.L., and Thomson G., 1994. Development and function of Pisolithus and Scleroderma ectomycorrhizas formed in vivo with Allocasuarina, Casuarina and Eucalyptus. Mycorrhiza 5: 129-138.

Gafur A., Schützendübel A., Langenfeld-Heyser R., Fritz E., and Polle A., 2004. Compatible and incompetent Paxillus involutus isolates for ectomycorrhiza formation in vitro with poplar (Populus $\times$ canescens) differ in $\mathrm{H}_{2} \mathrm{O}_{2}$ production. Plant Biol. 6: 91-99.

Gu R.S., Fonseca S., Puskas L., Hackler L., Zvara A., Dudits D., and Pais M.S., 2004. Transcript identification and profiling during salt stress and recovery of Populus euphratica. Tree Physiol. 24: 265-276.

Hampp R., and Schaeffer C., 1999. Mycorrhiza carbohydrate and energy metabolism. In: Varma A., Hock B., (Eds.) Mycorrhiza: structure, function, molecular biology and biotechnology. Springer-Verlag, Berlin, pp. 273-303.

Hampp R., Ecke M., Schaeffer C., Wallenda T., Wingler A., Kottke I., Sundberg B., 1996. Axenic mycorrhization of wild type and transgenic hybrid aspen expressing T-DNA indolacetic acid-biosynthesis genes. Trees (Berl.) 11: 59-64.

Holliman P.J., Clark J.A., Williamson J.C., and Jones D.L., 2005. Model and field studies of the degradation of cross-linked polyacrylamide gels used during the revegetation of slate waste. Sci. Total Environ. 336: $13-24$.

Hu L., Lu H., Liu Q., Chen X., and Jiang X., 2005. Overexpression of mtlD gene in transgenic Populus tomentosa improves salt tolerance through accumulation of mannitol. Tree Physiol. 25: 1273-1281.

Hukin D., Cochard H., Dreyer E., Le Thiec D., and Bogeat-Triboulot M.A., 2005. Cavitation vulnerability in roots and shoots: does Populus euphratica Oliv., a poplar from arid areas of Central Asia, differ from other poplar species? J. Exp. Bot. 56: 2003-2010.

Hüttermann A., Zommorodi M., and Reise K., 1999. Addition of hydrogels to soil for prolonging the survival of Pinus halepensis seedlings subjected to drought. Soil Till. Res. 50: 295-304.

IPCC, 2001. Climate Change. Cambridge University Press, Cambridge.

Kitajima M., and Butler W.L., 1975. Quenching of chlorophyll fluorescence and primary photochemistry in chloroplasts by dibromothyquinone. Biochim. Biophys. Acta 376: 105-115.

Landhausser S.M., Muhsin T.M., and Zwiazek J.J., 2002. The effect of ectomycorrhizae on water relations in aspen (Populus tremuloides) and white spruce (Picea glauca) at low soil temperatures. Can. J. Bot. 80: 684-689.

Langenfeld-Heyser R., Gao J., Ducic T., Tachd Ph., Lu C.F., Fritz E., Gafur A., and Polle A., 2007. Paxillus involutus mycorrhiza attenuate $\mathrm{NaCl}$-stress responses in the salt-sensitive hybrid poplar Populus $\times$ canescens. Mycorrhiza 17: 121-131.

Luo Z.B., Calfapietra C., Liberloo M., Scarascia-Mugnozza G., and Polle A., 2006. Carbon partitioning to mobile and structural fractions in poplar wood under elevated $\mathrm{CO}_{2}$ (EUROFACE) and $\mathrm{N}$-fertilization. Glob. Change Biol. 12: 272-283.
Marjanovic Z., Nehls U., and Hampp R., 2005. Mycorrhiza formation enhances adaptive response of hybrid poplar to drought. Ann. N. Y. Acad. Sci. 1048: 496-499.

Matzner E., Khanna P., Meiwes K., Lindheim M., Prenzel J., and Ulrich B., 1982. Elementflüsse in Waldökosystemen im Solling - Datendokumentation. Göttingen Bodenkundliche Berichte 71: $1-276$.

Merchant A., Tausz M., Arndt S.K., and Adams M.A., 2006. Cyclitols and carbohydrates in leaves and roots of 13 Eucalyptus species suggest contrasting physiological responses to water deficit. Plant Cell Environ. 29: 2017-2029.

Nehls U., Mikolajewski S., Magel E., and Hampp R., 2001. Carbohydrate metabolism in ectomycorrhizas: gene expression, monosaccharide transport and metabolic control. New Phytol. 150: 533-541.

Nehls U., Grunze N., Willmann M., Reich M., and Kuster H., 2007. Sugar for my honey: Carbohydrate partitioning in ectomycorrhizal symbiosis. Phytochemistry 68: 82-91.

Ottow E.A., Teichmann T., Kuhlmann B., Fritz E., Brosche M., Kangasjarvi J., Jiang X., and Polle A., 2005. Populus euphratica displays apoplastic sodium accumulation, osmotic adjustment by decreases in calcium and soluble carbohydrates, and develops leaf succulence under salt stress. Plant Physiol. 139: 1762-1772.

Polle A., Altman A., and Jiang X.N., 2006. Towards genetic engineering for drought tolerance in trees. In: Fladung M., Ewald D. (Eds.), Tree Transgenesis: recent developments. Springer Verlag, Berlin, pp. 275297.

Reddy M.S. and Satyanarayana T., 1998. Ectomycorrhizal formation in micropropagated plantlets of Populus deltoids. Symbiosis 25: 343-348.

Rennenberg H., Loreto L., Polle A., Brilli F., Fares S., Beniwal R.S., and Gessler A., 2006. Physiological responses of forest trees to heat and drought. Plant Biol. 8: 556-571.

Rincon A., Priha O., Lelu-Walter M.A., Bonnet M., Sotta B., and Tacon F.L., 2005. Shoot water status and ABA responses of transgenic hybrid larch Larix kaempferi $\mathrm{x}$ L. decidua to ectomycorrhizal fungi and osmotic stress. Tree Physiol. 25: 1101-1108.

Schär C., Vidale P.L., Lüthi D., Frei C., Häberli C., Mark A., Liniger M.A., and Appenzeller C., 2004. The role of increasing temperature variability in European summer heat waves. Nature 427: 332-336.

Sixto H., Aranda I., and Grau J.M., 2006. Assessment of salt tolerance in Populus alba clones using chlorophyll fluorescence. Photosynthetica 44: 169-173.

Smith S.E. and Read D.J., 1997. Mycorrhizal symbiosis. Cambridge, UK, Academic Press.

Vierheilig H., Alt M., Mohr U., Boller T., and Wiemken A., 1994. Ethylene biosynthesis and activities of chitinase and B-1,3-glucanase in the roots of host and non-host plants of vesicular-arbuscular mycorrhizal fungi after inoculation with Glomus mosseae. J. Plant Physiol. 143: 337-343.

Viero P.W.M. and Little K.M., 2006. A comparison of different planting methods, including hydrogels, and their effect on eucalypt survival and initial growth in South Africa. South. Afr. For. J. 208: 1-9.

Viero P.W.M., Little K.M., and Oscroft D.G., 2000. The effect of a soilamended hydrogel on the establishment of a Eucalyptus grandis $\times$ E. camaldulensis clone grown on the sandy soils of Zululand. South. Afr. For. J. 188: 21-28. 